\title{
The biomechanical influence of tibio-talar containment on stability of the ankle joint
}

\author{
Arno Frigg · Roman Frigg • Beat Hintermann • \\ Alexey Barg • Victor Valderrabano
}

Received: 25 January 2007/ Accepted: 30 May 2007/Published online: 13 July 2007

(C) Springer-Verlag 2007

\begin{abstract}
Chronic ankle instability (CAI) is a frequent sport orthopaedic entity. Although many risk factors have been studied extensively, little is known how it is influenced by the osseous joint configuration. Based on lateral $\mathrm{X}$-rays, the radius of the talar surface and the tibial coverage of the talus (sector $\alpha$ ) were measured on a DICOM/ PACS system in 52 patients with CAI and an age- and sexmatched control group. The talar radius was found to be larger in patients with CAI $(21.2 \pm 2.4 \mathrm{~mm})$ than in the control group $(17.7 \pm 1.9 \mathrm{~mm} ; P<0.0001)$. The tibio-talar sector was smaller in patients with CAI $\left(80^{\circ} \pm 5.1^{\circ}\right)$ than
\end{abstract}

ESSKA Award Paper for "Best Paper in Ligament and Biomechanics" (sponsored by Smith and Nephew) in Innsbruck, Austria 2006.

A. Frigg $\cdot$ V. Valderrabano

Department of Orthopaedic Surgery,

University of Basel, Basel, Switzerland

R. Frigg

London School of Economics, London, UK

B. Hintermann · A. Barg

Orthopaedic Clinic, Kantonsspital Liestal,

Liestal, Switzerland

A. Barg

Department of Applied Mathematics,

University of Münster, Münster, Germany

V. Valderrabano

Human Performance Laboratory,

University of Calgary, Calgary, Canada

A. Frigg $(\bowtie)$

Fichtenweg 7, 4127 Birsfelden, Switzerland

e-mail: mail@arnofrigg.com in the control group $\left(88.4^{\circ} \pm 7.2^{\circ} ; P<0.0001\right)$. The aim of this study is to analyse the biomechanical influence of the clinical data on stability of the ankle joint. A two-dimensional model of the tibio-talar joint in the sagittal plane was developed. The joint configuration was described by the tibio-talar sector $(\alpha)$ and the radius $(r)$ of the talus. The force $\left(F=F_{\mathrm{BW}} \tan \alpha / 2\right)$ and energy $\left(E=F_{\mathrm{BW}} r[1-\cos \alpha /\right.$ 2]) to dislocate the talus out of the tibial plafond were deduced. Ankle stability is a function of the tibio-talar sector: the force necessary to dislocate the joint is decreasing with a smaller sector. The clinical data show that the force needed to dislocate the ankle of CAI patients was $14 \%$ weaker than the one needed in the case of healthy subjects $(P<0.0001)$. The energy to dislocate the ankle depends both on the sector and the radius. The clinical data do not show a significant difference between the energy needed to dislocate the joint of CAI patients and the one of healthy subjects. This is because there is a correlation of a small sector and a large radius for CAI ankles. CAI is associated with an unstable osseous joint configuration, which is characterized by a larger radius of the talus and a smaller tibio-talar sector. The findings of the biomechanical model explain the clinical observations and demonstrate how stability of the ankle joint is influenced by the osseous configuration. Surgical ankle ligament stabilization might be more recommended in patients with an unstable osseous configuration as such patients have a disposition for recurrent sprains. Removing anterior osteophytes for anterior impingement should be done carefully in CAI patients because this would decrease the tibial coverage of the talus and thus dispose the talus to dislocate anteriorly. People who have an unstable ankle configuration and who nevertheless engage in activities with high risk of ankle sprains could be asked to wear ankle protecting sports equipment. 
Keywords Ankle instability · Biomechanics · Osseous configuration $\cdot$ Ligaments

\section{Introduction}

Ankle sprains are the most frequent injuries in sports and recreational activities [12]. Eighty-five percent of sprains affect the anterior talo-fibular ligament (ATFL) [1, 13, 34] and $20-40 \%$ of patients develop chronic ankle instability (CAI) $[4,6,9,16]$. According to Beynnon, the risk factors for ankle sprains can be divided in intrinsic factors (hindfoot alignement, ligament laxity, neuromuscular control etc.) and extrinsic factors (shoes, type and intensity of sports, warm up etc.) [2, 14]. It is known, that the shoulder, a joint characterized by a large humeral head compared to a small glenoid, is a rather unstable joint whereas the hip, a joint characterized by a large coverage of the femoral head by the acetabulum, is a rather stable joint. We therefore suggest that there must also be a connection between the osseous joint configuration of the ankle and ankle stability.

We compared 52 patients with CAI (18 males and 34 females, average age $39 \pm 13.9$ years), who suffered from at least three recurrent sprains, with an age- and sex matched control group of 52 healthy subjects (18 males and 34 females, average age $37 \pm 16.5$ years) [10]. Using a DICOM/PACS application, lateral radiographs of the ankle were analyzed by a blinded radiologist. The following parameters were measured [23]: (1) The radius of the talus $(r)$ by digitally fitting a circle to the talar joint surface and then determining the centre of talus; (2) the tibio-talar sector $(\alpha)$ by measuring the angle between the lines drawn from the centre of the talus to the anterior and posterior margins of the distal tibia.

The clinical results show, that patients with CAI had a significantly larger talar radius $(21.2 \pm 2.4 \mathrm{~mm})$ than healthy subjects $(17.7 \pm 1.9 \mathrm{~mm} ; P<0.0001$, Power $>95 \%$; Fig. 1; Table 1). Patients with CAI had also a significantly smaller tibio-talar sector $\left(80^{\circ} \pm 5.11^{\circ}\right)$ than normal subjects $\left(88.4^{\circ} \pm 7.2^{\circ} ; P<0.0001\right.$, Power $>95 \%$; Fig. 1; Table 1) [10]. We therefore concluded that the osseous configuration of the ankle joint is another intrinsic risk factor contributing to ankle instability.

This raises the question of why a rather small anatomical difference (a 3.5-mm larger radius and a $8.4^{\circ}$ smaller sector) can mark a distinction between stable and unstable ankles.

We answer this question by dint of a mechanical model of the ankle joint, which allows a biomechanical analysis of the clinical-anatomical data on forces and energies needed to dislocate stable and unstable joints.
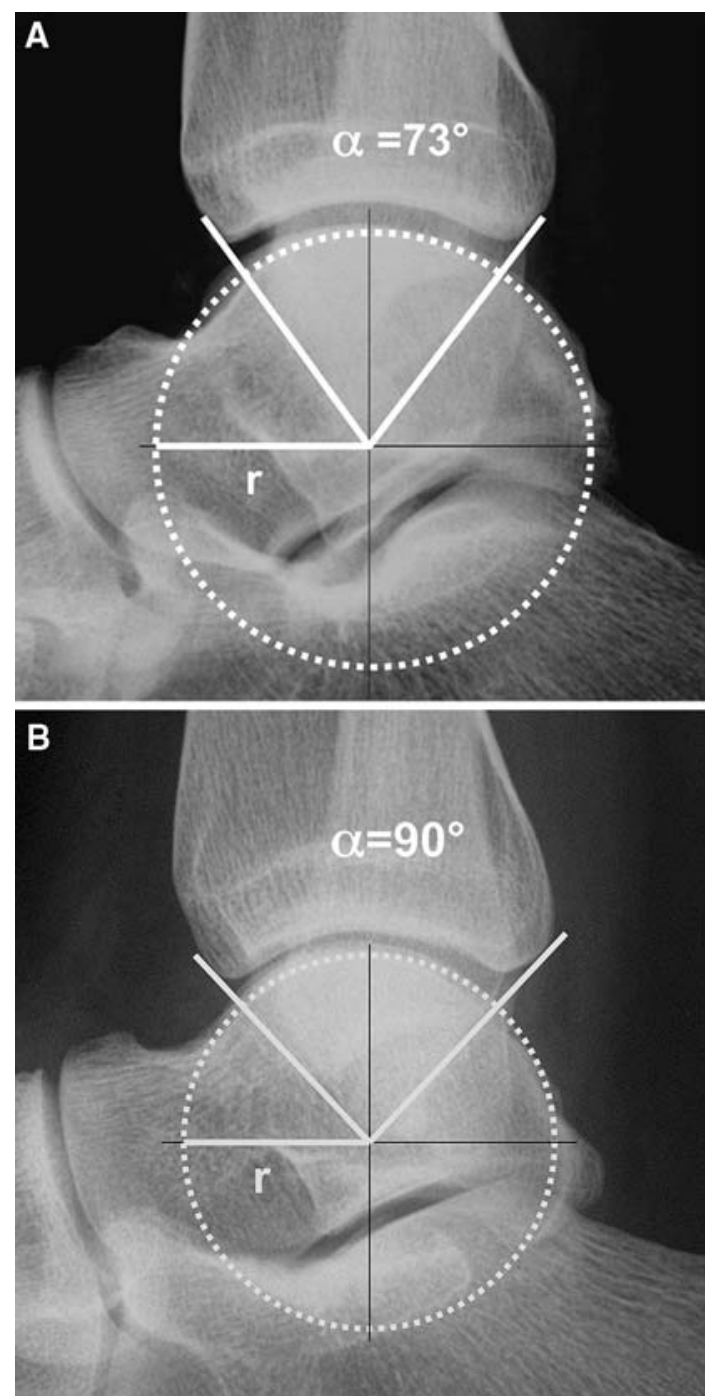

Fig. 1 Radiographs of a typical unstable (a) and stable (b) ankle joint configuration

Table 1 Results

\begin{tabular}{lllll}
\hline & & CAI patients & Healthy subjects & $P$ \\
\hline Radius $(r)$ & Mean & $21.2 \mathrm{~mm}$ & $17.7 \mathrm{~mm}$ & $<0.0001$ \\
& Range & $17-26 \mathrm{~mm}$ & $13-23 \mathrm{~mm}$ & \\
& SD & 2.4 & 1.9 & \\
Sector $(\alpha)$ & Mean & $80.0^{\circ}$ & $88.4^{\circ}$ & $<0.0001$ \\
& Range & $72^{\circ}-95^{\circ}$ & $79^{\circ}-111^{\circ}$ & \\
& SD & 5.1 & 7.2 & $<0.0001$ \\
$F_{\mathrm{L}}$ & Mean & $0.84 F_{\mathrm{BW}}$ & $0.98 F_{\mathrm{BW}}$ & \\
& SD & 0.08 & 0.13 & 0.66 \\
$E_{\mathrm{L}}$ & Mean & $0.0049 \mathrm{~J} / \mathrm{N}$ & $0.005 \mathrm{~J} / \mathrm{N}$ & \\
& SD & 0.00058 & 0.00073 &
\end{tabular}

$C A I$ chronic ankle instability, $S D$ standard deviation, $F_{\mathrm{L}}$ luxation force, $E_{\mathrm{L}}$ luxation energy 


\section{Materials and methods}

According to the clinical situation with measurement of lateral radiographs of the ankle, a simple two-dimensional model of the ankle in the sagittal plane was used. The biomechanical model is based on the assumption that the ankle joint is spherical and that there is no friction between talus and tibia. The calculations were performed in a plantigrade ankle joint position and in dorsi- and plantarflexion $(\gamma=$ angle of the tibia to neutral position of the joint perpendicular to the ground). Further, the model only considers the osseous configuration and neglects the ligaments and muscle restrain forces.

The center of the talus is defined as the center of a circle fitted to the talar surface. The radius $(r)$ is defined as the distance between the centre of the talus and the periphery. The tibio-talar sector $(\alpha)$ is defined as the angle between the two lines drawn from the center of the talus to the anterior and posterior margins of the tibia (Fig. 1).

The depth of the tibial plafond $(h)$ was defined as the height of the semicircle above the joint line (Fig. 5a). Two forces act on the joint: The body weight force $\left(F_{\mathrm{BW}}\right)$ acting vertically, pressing the tibia onto the talus, and an external anteriorly orientated luxation force $F_{\mathrm{L}}$ caused by a anteriorly oriented sprain, acting in an angle $\delta$ to the ground (Fig. 2). $F_{\mathrm{L}}$ and $F_{\mathrm{BW}}$ are force vectors, for typographical simplicity we have omitted the arrows on top of the letter symbols).

Force to unlock the ankle joint

\section{Position perpendicular to the ground}

First the force needed to "unlock" the ankle joint is determined; that is, the force necessary to bring the joint from the state of full joint contact with force transmission through the entire joint surface (Fig. 2a) to the state of minimal dislocation with force transmission through the anterior margin of the tibia (Fig. 2b).

Figure 2 shows a lateral view of the loaded ankle joint. When an anteriorly orientated luxation force $\left(F_{\mathrm{L}}\right)$ acts on the talus, as is often the case in an acute ankle sprains, forces are created at the anterior margin of the tibia because of its concave articular surface. The tibia will stay on the talus (e.g. the talus will not be able to dislocate out of the tibia) as long as force $F_{\mathrm{LT}}$ (tangential component of luxation force $F_{\mathrm{L}}$ ) is smaller or equal to the counteracting force $F_{\mathrm{BWT}}$ (tangential component of $F_{\mathrm{BW}}$ ). If $F_{\mathrm{LT}}$ becomes larger than $F_{\mathrm{BWT}}$, the tibia will dislocate. Hence, the magnitude of the minimal force that is needed to dislocate the ankle is obtained as the solution of the equation:

$\left|F_{\mathrm{LT}}\right|=\left|F_{\mathrm{BWT}}\right|$
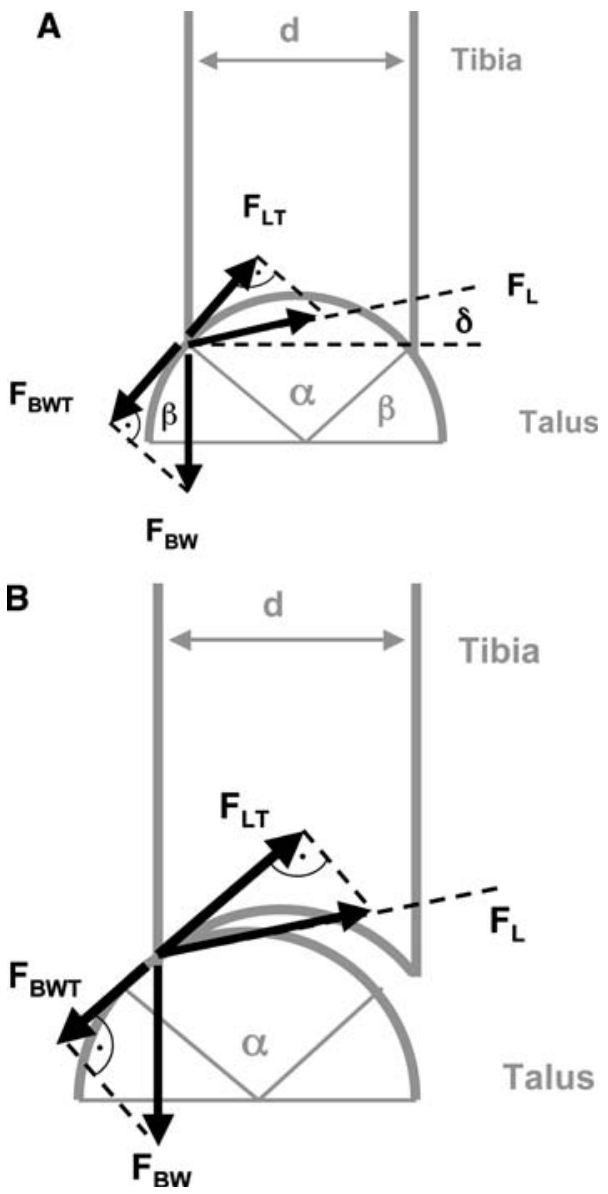

Fig. 2 Biomechanical model: $\alpha$ sector of tibial coverage of the talus; $\beta(180-\alpha) / 2 ; \delta$ angle of $F_{\mathrm{L}}$ to the ground; $d$ width of tibia at the level of the joint. a Forces in the undislocated ankle joint: $F_{\mathrm{BW}}$ body weight force; $F_{\mathrm{BWT}}$ tangential component of $F_{\mathrm{BW}}\left(=\cos \beta F_{\mathrm{BW}}\right) ; F_{\mathrm{L}}$ luxation force acting to the tibia; $F_{\mathrm{LT}}$ tangential component of $F_{\mathrm{L}}\left[=F_{\mathrm{L}} \cos \right.$ $(90-\beta-\delta)]$. b Dislocated ankle joint: $F_{\mathrm{LT}}>F_{\mathrm{BWT}}$ and $F_{\mathrm{BWT}}$ getting smaller with further dislocation

First, $\left|F_{\mathrm{LT}}\right|$ and $\left|F_{\mathrm{BWT}}\right|$ are expressed as functions of the basic parameters as shown in Fig. 2: geometrical considerations on the basis of Fig. 2 yield that $\left|F_{\mathrm{BWT}}\right|=\left|F_{\mathrm{BW}}\right|$ $\cos \beta$ and

$\left|F_{\mathrm{LT}}\right|=\left|F_{\mathrm{L}}\right| \cos (90-\beta-\delta)$. Hence the basic equation becomes:

$\left|F_{\mathrm{BW}}\right| \cos \beta=\left|F_{\mathrm{L}}\right| \cos (90-\beta-\delta)$

After some calculations, the solution is (for all $\delta \geq 0$ ):

$\left|F_{\mathrm{L}}\right|=\left|F_{\mathrm{BW}}\right| \frac{\sin \frac{\alpha}{2}}{\cos \left(\frac{\alpha}{2}-\delta\right)}$.

In the special case of $\delta=0$ (e.g. a horizontal luxation force) the equation reduces to

$\left|F_{\mathrm{L}}\right|=\left|F_{\mathrm{BW}}\right| \tan \left(\frac{\alpha}{2}\right)$ 
The graph of this function is shown in Fig. 3. It shows that the smaller the sector angle $\alpha$ is, the smaller the force needed to dislocate the tibia is. This means that, with regard to the osseous configuration, joints with small $\alpha$ are less stable than joints with large $\alpha$. For $\alpha=0^{\circ}$ the articular surfaces would not be able to provide any stability for the joint; whereas for $\alpha=180^{\circ}$ the force to dislocate the ankle would become infinite.

Another aspect is the magnitude of the radius of talus. If the width of the tibia $(d)$ would not vary (as shown in Fig. $5 \mathrm{a}+\mathrm{c}$ ), a larger radius implies a smaller tibial coverage angle $(\alpha)$ of the talus and therefore contributes to instability.

\section{Position in flexion and extension}

The considerations have been made so far for the ankle in neutral position, where the tibia is perpendicular to the ground. This is, however, not the case during gait and sports activities. If the foot is moved into dorsiflexion and the tibia into anteroflexion, respectively, the effective tibial coverage of talus becomes larger (Fig. 4a); whereas if the foot is moved into plantar flexion, and the tibia into retroflexion respectively, the effective tibial coverage of talus becomes smaller (Fig. 4b). Assuming the tibia is angulated by an angle $\gamma$ to the perpendicular axis to the ground (tibia in anteroflexion, positive value; tibia in retroflexion, negative value), the force required to dislocate the tibia is: (for all $\delta \geq 0$ and $\gamma$ between $-\alpha / 2$ and $90-\alpha / 2$ ):

$\left|F_{\mathrm{L}}\right|=\left|F_{\mathrm{BW}}\right| \frac{\sin \left(\frac{\alpha}{2}+\gamma\right)}{\cos \left(\frac{\alpha}{2}+\gamma-\delta\right)}$

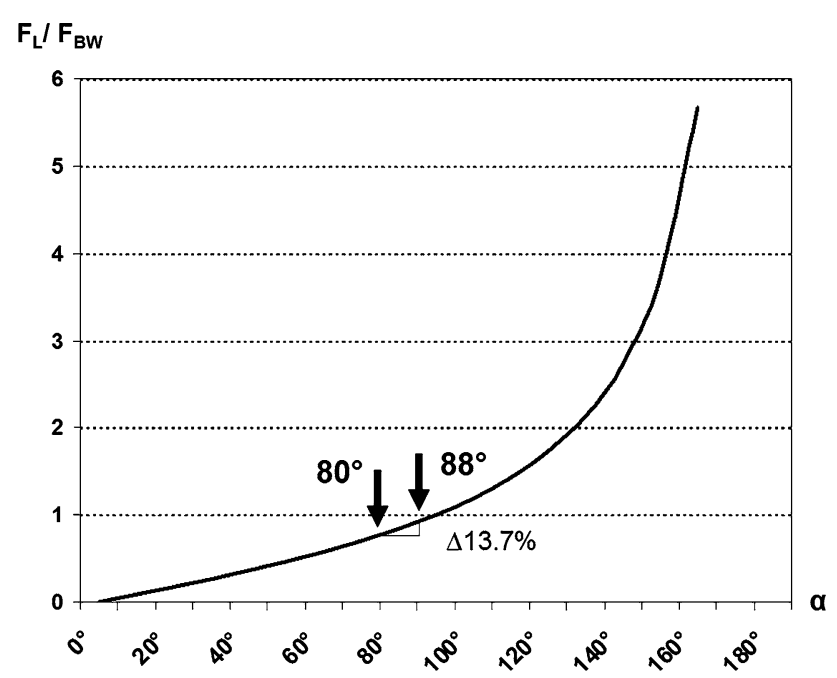

Fig. 3 Force $\left(F_{\mathrm{L}}\right)$ needed per body weight force $\left(F_{\mathrm{BW}}\right)$ to unlock the ankle depending on the tibio-talar sector $\left(F=F_{\mathrm{BW}} \tan \alpha / 2\right)$. Clinical implication When applying this model to the clinical findings (CAI patients with $\alpha=80^{\circ} \pm 5.11^{\circ}$, healthy subjects with $\alpha=88.4^{\circ} \pm 7.2^{\circ}$ ), the force $F_{\mathrm{L}}$ required to dislocate the ankle is $13.7 \%$ lower in the subjects with CAI than for healthy subjects
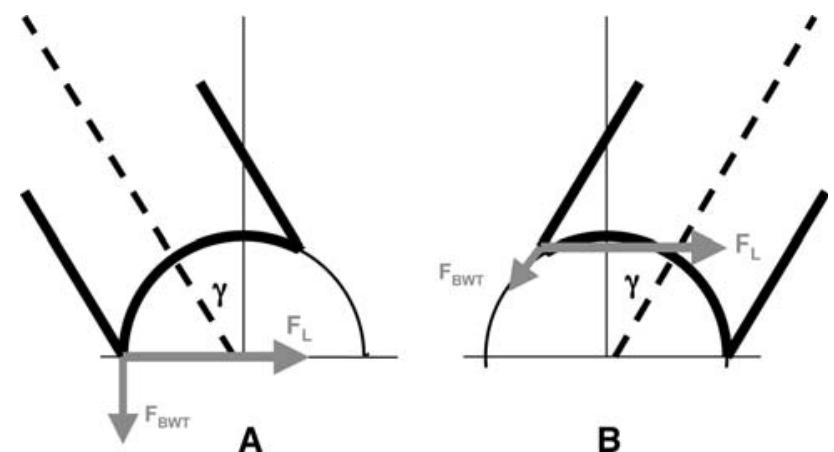

Fig. 4 Force needed to unlock the ankle depending on different joint positions $(\gamma=$ angle of the tibia to neutral position of the joint perpendicular to the ground): $\mathbf{a}$ in dorsiflexion the ankle becomes more stable; $\mathbf{b}$ while in plantar flexion the ankle becomes more unstable

In the special case of $\delta=0$ (e.g. a horizontal luxation force) the Eq. (4) can be reduced to:

$\left|F_{\mathrm{L}}\right|=\left|F_{\mathrm{BW}}\right| \tan \left(\frac{\alpha}{2}+\gamma\right)$

This force exhibits the same qualitative behaviour as that one shown in neutral position: In plantarflexion, the joint gets less stable due to the decrease in anterior tibial coverage.

Energy required for dislocating the tibia

\section{Position perpendicular to the ground}

Another aspect of interest is the energy that is required to completely dislocate the loaded ankle, i.e. to lift the margin of the tibia on top of the talus (Fig. 5). Assuming again that $\gamma=0$ (i.e. that the tibia is perpendicular to the ground), the calculation shows that the dislocation energy $E_{\mathrm{L}}$ is given by (Fig. 5):

$E_{\mathrm{L}}=\left|F_{\mathrm{BW}}\right| r\left[1-\cos \left(\frac{\alpha}{2}\right)\right]$.

This function is plotted in Fig. 6. The more energy is required to dislocate the ankle, the more stable the joint is. This means, again, that a smaller the tibio-talar sector leads to a less stable joint configuration.

\section{Position in flexion and extension}

Finally, the situation in which the tibia does not need to be perpendicular to the ground is considered (Fig. 7). The energy needed to lift the tibia reaches a maximum for dorsiflexion of the talus and anteroflexion of the tibia, and minimum for plantar flexion of the talus and retroflexion of 


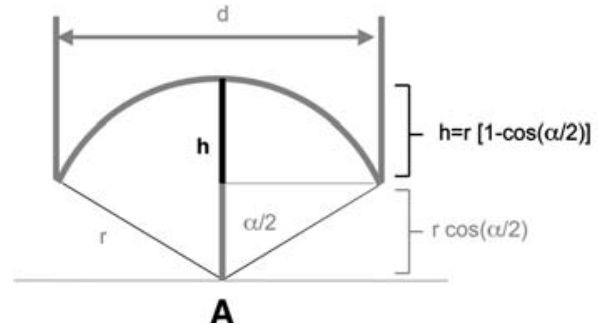

Fig. 5 Biomechanical model of the ankle: a A small radius $(r)$ of the talus results in a wide sector $(\alpha)$ and a deep cavity of tibial plafond $(h)$, which means high intrinsic stability of the joint due to optimal containment. b With a constant radius, a smaller sector implies a smaller depth of the tibial plafond $(h)$ making the ankle more
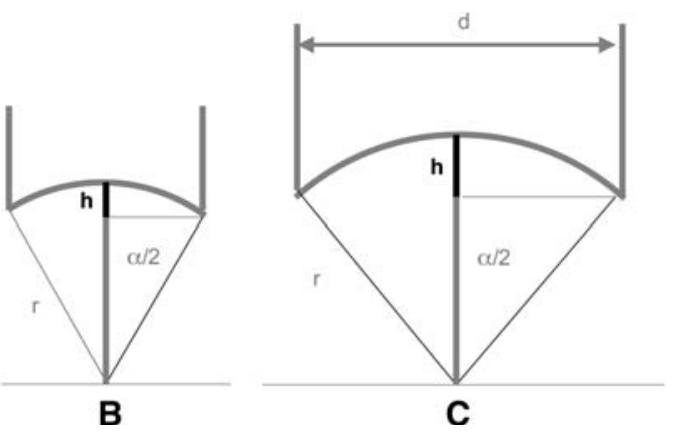

unstable. c With a constant width of the tibia $(d)$, a larger radius results in a smaller sector angle $(\alpha)$ and a smaller cavity of the tibial plafond $(h)$, which means less intrinsic stability of the joint due to minimal containment

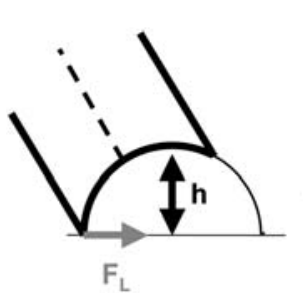

A Dorsiflexion

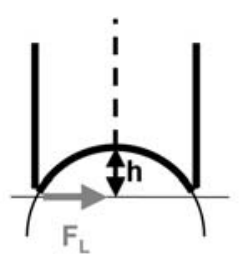

B Foot on ground

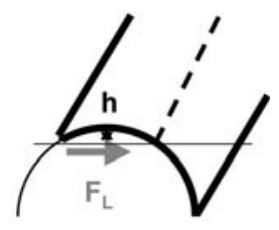

C Plantar flexion
Fig. 7 Energy needed to lift the tibia depending on the depth $(h)$ of the tibial plafond is highest for dorsiflexion of the talus and anteroflexion of the tibia (a), less for neutral position (b), and is minimal for plantar flexion of the talus and retroflexion of the tibia, respectively (c)

$P \leq 0.05$. All statistical calculations were performed using the STATISTICA statistical package (Version 6.1, USA, 2003).

talus depending on the tibio-talar sector $\left[E_{\mathrm{L}}=F_{\mathrm{BW}} r(1-\cos \alpha / 2)\right.$ for a constant radius. Clinical implication: When applying this model to the clinical findings (CAI patients $\alpha=80^{\circ} \pm 5.11^{\circ}$, healthy subjects $\alpha=88.4^{\circ} \pm 7.2^{\circ}$ ) the energy needed to dislocate the ankle is, for a constant radius, $17.4 \%$ lower in the subjects with ankle instability than for subjects with a stable ankle. With inclusion of the clinically measured radius (CAI patients $r=21.2 \pm 0.24 \mathrm{~mm}$, healthy subjects $r=17.7 \pm 1.9 \mathrm{~mm}$ ) the smaller sector seems to be neutralized by the larger radius; therefore no statistical significant difference in the depth of the tibial plafond and the energy could be measured

the tibia respectively. In this case the dislocation energy is given by

$E_{\mathrm{L}}=\left|F_{\mathrm{BW}}\right| r\left[1-\cos \left(\frac{\alpha}{2}+\gamma\right)\right]$.

\section{Statistical methods and data analysis}

Statistical differences among groups were determined by the unpaired student's $t$ test. Significance was considered at

\section{Results}

Calculations with the clinically determined values (Table 1) have shown that the luxation force $F_{\mathrm{L}}$ for unstable configured ankles is significantly lower $\left(0.84 F_{\mathrm{BW}}, \mathrm{SD} 0.08\right)$ than for stable ankles $\left(0.98 F_{\mathrm{BW}}\right.$, SD $0.13 ; P<0.0001$; Table 1$)$. Hence, a $13.7 \%$ weaker force is needed to dislocate the clinically unstable configured ankle.

The energy needed to dislocate the ankle, for a constant radius, is $17.4 \%$ lower for subjects with ankle instability $(0.24 \mathrm{~J} / \mathrm{N}, \mathrm{SD} 0.029)$ than for subjects with a stable ankle $(0.28 \mathrm{~J} / \mathrm{N}, \mathrm{SD} 0.045 ; P<0.0001)$.

In the clinical data, there is a negative correlation of radius and sector (correlation coefficient $-0.64, P<0.05$ ). This means that the typical configuration of CAI ankle is charaterized by a small sector and a large radius, whereas the typical stable ankle is charaterized by a large sector and 
small radius. Theoretically, also a combination of a large radius and large sector or a small radius and small sector would be possible, but have not been observed. This may be explained by the fact that if the width of the tibia $(d)$ would not vary, a larger radius implies a smaller sector $(\alpha)$, and therefore is associated with instability (Fig. 5). With inclusion of both the clinically measured radius and sector, no statistically significant difference in the depth of the tibial plafond and the energy could be measured (Table 1), because the decrease in energy caused by the smaller sector seems to be neutralized by the increase in radius.

\section{Discussion}

This study demonstrates how ankle stability is influenced biomechanically by the osseous configuration of the ankle joint and it explains the clinical observation that a smaller tibio-tallar sector and larger radius are associated with CAI. The biomechanical model shows that ankle stability is predominantly a function of the tibio-talar sector. In particular, the force $F_{\mathrm{L}}$ needed for ankle dislocation decreases with a smaller sector $\left(F_{\mathrm{L}}=F_{\mathrm{BW}} \tan \alpha / 2\right)$. Fourteen percent less force is needed to dislocate the ankle joint of CAI patients compared to healthy subjects. One would expect a similar relation between the energy needed for dislocation and CAI patients. However, such a relation is not observed clinically because the energy $E_{\mathrm{L}}$ to dislocate the ankle depends both on the sector and the radius $\left(E_{\mathrm{L}}=F_{\mathrm{BW}} r\right.$ $[1-\cos \alpha / 2])$ and because of the clinical association of a small sector with a large radius in CAI ankles, no significant difference in the energy could be found with the clinical data even though in the biomechanical model the energy is decreasing with a smaller sector. This study contributes to the understanding of ankle instability and explains why some CAI patients suffer from recurrent sprains (even though they have previously undergone surgical ligament repair $[16,28])$ by appeal to geometrical features of their osseous joint configurations.

The usually described injury mechanism of an ankle sprain (a rollover over the plantarflexed inverted foot after heelstrike [5]) is in accordance with our model: the normal gait pattern consists of heel strike, plantarflexion, dorsalextension, heel rise and toe off [11]. The phase of plantarflexion is described as most vulnerable phase for an ankle sprain $[5,11]$. This observation is consistend with our model because in plantarflexion the anterior tibio-talar sector gets minimal and the required luxation force decreases (Fig. 4).

In our model, we have hypothesized that the axial load increases ankle stability while the tibia is pressed onto the talus by the body weight. This observation was also made by others: $84-94 \%$ of the body weight is transmitted through the tibio-talar joint, and only $6-16 \%$ is loaded on the fibula [5]. Fraser and Ahmed [8] studied the passive rotational stability of the loaded ankle and found that with increasing load rotation decreased. McCullough and Burge [24] found too that increasing the axial load decreased the axial rotation in the ankle with intact ligaments as well as after ligament release. Stormont et al. [30], in a preloaded model, found that the articular surfaces supplied $30 \%$ of the stability in rotation and $100 \%$ of the stability in inversion testing.

\section{Clinical implication}

The observation, that a smaller tibio-talar sector is associated with CAI has several clinical implications: (1) Surgical ankle ligament stabilization might be earlier recommended in patients with an unstable osseous configuration, because such patients have a disposition for recurrent sprains. Such a treatment seems particularly important in these cases because CAI might lead to posttraumatic ligamentous ankle osteoarthritis [32]. (2) In case of impingement, removing the osteophytes at the anterior tibia is widely recommended in the literature [3, 33]. However, this treatment would destabilize the ankle joint, and should be done with caution in case of patients with previous sprains. (3) This study might be of great importance for the prevention of ankle ligament injuries in sports. People engaging in activities with high risk for ankle sprains (e.g. basketball, football, soccer) and who have an unstable ankle configuration should be asked to wear ankle protecting sports equipment $[25,26]$. (4) In case of an unstable joint configuration there will be generally more stress on the ligaments, leading to ligament laxity and consequently CAI.

\section{Limitations of the study}

The ankle joint is a complex joint with wider anterior talus, larger lateral talar radius, a larger posterior talar radius, and larger tibial radius than talar radius, a moving center of rotation and it consists of three bones, which leads to a complex three-dimensional motion pattern [15, 18, 20, 21]. The motion is predominantly sagittal but not purely hinged because dorsiflexion yields slight external rotation whereas plantar flexion causes slight internal rotation [5]. This is why the question arises whether the ankle joint can be adequately described in a two-dimensional model. Several points support this simplifaction. (1) Eighty-five percent of sprains affect the ATFL, representing a luxation movement in the antero-posterior direction [1, 13, 34]. (2) From both experimental and mathematical studies, Leardini et al. [17] have concluded that the human ankle joint can be modelled in the sagittal plane. Approximating the ankle joint as a 
simple hinge joint has been accepted and many models are based on this approximation [18, 29]. (3) Also other studies have focused on the sagittal plane only. Tochigi et al. [31] found that an anterior or posterior positioning of the talus in the mortise increases mechanical stress and therefore leads to osteoarthritis. Lebrun et al. [19] found that a posterior position of the fibula is predisposing for ankle instability. (4) Our model is based on the clinical situation with lateral radiographs and allows to make clinical statements simply by using a lateral radiographs. Although our model makes significant idealisations, it corresponds to clinical observations and allows us to explain the clinical findings. (5) The joint movement could be completely described by movements and rotations in three dimensions. While frontal and axial movements would lead to fracture, sagittal-movement would lead to luxation. While a rotation in the saggital plane describes the normal joint movement, a transversal rotation is only normal up to $10^{\circ}$ and a frontal rotation up to $5^{\circ}$ [22]; further rotation would cause fracture. Compared to the $70^{\circ}-90^{\circ}$ of flexion/extension, the axial and lateral rotations have only a small influence and therefore have been neglected in this study.

We assumed no friction between talus and tibia, therefore we did not determine the work to move the tibia in posterior direction. In case of friction, the work for this posterior movement could be calculated by integration of equation (4), but because the friction in joints is minimal ( $\mu=0.005-0.04$ [7]), the influence on the obtained results is negligible.

The role of ligaments and muscles has been neglected in this study. In reality, the forces to dislocate the ankle would therefore be higher. Moreover, in normal gait, the joint reaction force can be as high as four times the body weight, which leads to another increase in the luxation force [27].

\section{Conclusions}

CAI is associated with an unstable osseous joint configuration, which is characterized by a larger radius of the talus, corresponding to a flatter talus and a smaller tibio-talar sector, which eventually leads to a smaller tibio-talar containment. The biomechanical model showed that stability is predominantly a function of the tibio-talar sector. The force $F_{\mathrm{L}}$ needed for ankle dislocation is decreasing with a smaller sector $\left(F_{\mathrm{L}}=F_{\mathrm{BW}} \tan \alpha / 2\right)$; in fact, a $14 \%$ weaker force is needed to dislocate the ankle joint of CAI patients compared to healthy subjects. The energy $E_{\mathrm{L}}$ to dislocate the ankle is depending both on the sector and the radius $\left[E_{\mathrm{L}}=F_{\mathrm{BW}} r(1-\cos \alpha / 2)\right]$. The clinical data do not show a significant difference in the energy in CAI patients compared to healthy subjects because there is a clinical correlation of a small sector and a large radius for CAI ankles.

\section{References}

1. Baumhauer JF, Alosa DM, Renstroem PA (1995) A prospective study of ankle injury risk factors. Am J Sports Med 23:564-570

2. Beynnon BD (2002) Predictive factors for lateral ankle sprains: a literature review. J Athl Train 37:376-380

3. Biedert R (1991) Anterior ankle pain in sport medicine: etiology and indications for arthroscopy. Arch Orthop Traum Surg 110:293-297

4. Brand RL, Collins MD, Templeton T (1981) Surgical repair of ruptured lateral ankle ligaments. Am J Sports Med 9:40-44

5. Brinker MR (2001) Review of orthopaedic trauma. Saunders, Philadelphia

6. Brostroem L (1966) Sprained ankle V-treatment and prognosis. Acta Chir Scand 132:537-550

7. Buckwalter JA, Einhorn TA, Simon SR (2000) Orthopaedic basic science, 2nd edn. AAOS, Rosemont

8. Fraser GA, Ahmed AM (1983) Passive rotational stability of the weight-bearing talocrural joint: an in vitro biomechanical study. Orthop Trans 7:248

9. Freeman MA (1965) Instability of the foot after injuries to the lateral liagment of the ankle. J Bone Joint Surg 47B:669-677

10. Frigg A, Magerkurth O, Valderrabano V, Ledermann HP, Hintermann B (2007) The effect of osseous ankle configuration on chronic ankle instability. Br J Sports Med (in press). doi:10.1136/ bjsm.2006.032672

11. Gage JR (1990) An overview of normal walking. Instr Course Lect 39:291-303

12. Garrick JG (1977) The frequency of injury mechanism of injury and epidemiology of ankle sprains. Am J Sports Med 5:241-142

13. Giza E, Fuller C, Junge A et al (2003) Mechanisms of foot and ankle injuries in soccer. Am J Sports Med 31:550-554

14. Halasi T, Kynsburg A, Tallay A (2004) Development of a new activity score for the evaluation of ankle instability. Am J Sports Med 32:899-908

15. Hintermann B, Nigg BM (1995) In vitro kinematics of the axially loaded ankle complex in response to dorsiflexion and plantarflexion. Foot Ankle Int 16:514-518

16. Karlsson J, Bergstern T, Peterson L (1988) Reconstruction of the lateral ligaments of the ankle for chronic lateral instability. J Bone Joint Surg 70A:581-588

17. Leardini A, O'Connor JJ, Catani F et al (1999) A geometric model of the human ankle joint. J Biomechanics 32:585-591

18. Leardini A, O'Connor JJ, Catani F et al (1999) Kinematics of the human ankle complex in passive flexion; a single degree of freedom system. J Biomechanics 32:111-118

19. LeBrun CT, Krause JO (2005) Variations in mortise anatomy. Am J Sports Med 33:852-855

20. Lundberg A, Svennson OK, Nemeth G et al (1989) The axis of rotation of the ankle joint. J Bone Joint Surg 71B:94-99

21. Macko VW, Matthews LS, Zwirkoski P (1991) The joint-contact area of the ankle. J Bone Joint Surg 73B:347-351

22. MacWilliams BA, Cowley M, Nicholson DE (2003) Foot kinematics and kinetics during adolescent gait. Gait Posture 17:214-224

23. Magerkurth O, Knupp M, Ledermann H et al (2006) Evaluation of hindfoot dimensions: a radiological study. Foot Ankle Int 27:612-616

24. McCullough CJ, Burge PD (1980) Rotatory stability of the loadbearing ankle. An experimental study. J Bone Joint Surg 62B:460-464

25. Müller CC, Hintermann B (1996) Die Wirkung von äusseren Stabilisierungshilfen auf die Rotationsinstabilität des Sprunggelenks. Sportverletzung Sportschaden 20:84-87

26. Nigg BM, Nurse MA, Stefanyshyn DJ (1999) Shoe inserts and orthotics for sport and physical activities. Med Sci Sports Exerc $31: 421-428$ 
27. Prochter P, Paul JP (1982) Ankle joint biomechnics. J Biomech 15:627-634

28. Rosenbaum D, Becker HP, Sterk J et al (1997) Functional evaluation of the 10-year outcome after modified Evans repair for chronic ankle instability. Foot Ankle Int 18:765-771

29. Stagni R, Leardnini A, Ensini A et al (2005) Ankle morphometry evaluated using a new semi-automated techinque based on X-ray pictures. Clinical Biomechnics 20:307-311

30. Stormont DM, Morrey BF, An KN et al (1985) Stability of the loaded ankle. Am J Sports Med 13:295-300

31. Tochigi Y, Suh JS, Amendola A et al (2006) Ankle alignement on lateral radiographs. Part 2: reliability and validity of measures. Foot Ankle Int 27:88-92
32. Valderrabano V, Hintermann B, Horisberger M et al (2006) Ligamentous posttraumatic ankle osteoarthritis. Am J Sports Med 34:612-620

33. Van Dijk CN, Tol JL, Verheyen CC (1997) A prospective study of prognostic factors concerning the outcome of arthroscopic surgery for anterior ankle impingement. Am J Sports Med 25:737-745

34. Wright IC, Neptune RR, van den Bogert AJ et al (2000) The influence on foot positioning on ankle sprains. J Biomech 33:513-519 